\title{
Radiation dose to the heart with hypofractionation in patients with left breast cancer
}

\author{
Dr. Budhi Singh Yadav, $\mathrm{MD}^{1 *}$ (D), Dr. Deepak Kumar Das, $\mathrm{MD}^{2}$, Dr. Narendra Kumar, $\mathrm{MD}^{3}$, \\ Dr. Manphool Singhal, $\mathrm{MD}^{4}$ and Mr. Ngangom Robert, $\mathrm{Phd}^{5}$ \\ ${ }^{1}$ Additional Professor, Radiation Oncology, PGIMER, Sector - 12, Chandigarh, India, ${ }^{2}$ Senior resident, Department of \\ Radiotherapy, AIIMS, Bhubaneswar 751019, India, Email ID- deepak.scb2006@gmail.com, ${ }^{3}$ Professor, Department of \\ Radiotherapy, PGIMER, Chandigarh 160012, India, Email ID- drnarendra74@gmail.com, ${ }^{4}$ Additional Professor, Department \\ of Radiodiagnosis, PGIMER, Chandigarh 160012, India, Email ID- drmsinghal74@gmail.com, and ${ }^{5}$ Medical physicist, \\ Department of Radiotherapy, PGIMER, Chandigarh 160012, India, Email ID- ngangom.robert@yahoo.com \\ ${ }^{\star}$ Corresponding Author. E-mail: drbudhi@gmail.com
}

(Received 12 February 2021; Revised 15 May 2021; Accepted 17 May 2021)

\begin{abstract}
Introduction: In this study we compared radiation dose received by organs at risk (OARs) after breast conservation surgery(BCS) and mastectomy in patients with left breast cancer.

Materials and methods: Total 30 patients, 15 each of BCS and mastectomy were included in this study. Planning Computerised Tomography (CT) was done for each patient. Chest wall, whole breast, heart, lungs, $\mathrm{LAD}$, proximal and distal $\mathrm{LAD}$, and contra lateral breast was contoured for each patient. Radiotherapy plans were made by standard tangent field. Dose prescribed was $40 \mathrm{~Gy} / 16 \# / 3$ weeks. Mean heart dose, LAD, proximal and distal LAD, mean and V5 of right lung, and mean, V5, V10 and V20 of left lung, mean dose and V2 of contra lateral breast were calculated for each patient and compared between BCS and mastectomy patients using student's $\mathrm{T}$ test.

Results: Mean doses to the heart, LAD, proximal LAD and distal LAD were 3.364Gy, 16.06Gy, 2.7Gy, 27.5Gy; and 4.219Gy, 14.653Gy, 4.306Gy, 24.6Gy, respectively for mastectomy and BCS patients. Left lung mean dose, V5, V10 and V20 were $5.96 \mathrm{~Gy}, 16 \%, 14 \%, 12.4 \%$; and $7.69 \mathrm{~Gy}, 21 \%, 18 \%$ and $16 \%$ in mastectomy and BCS patients, respectively. There was no statistical significant difference in the doses to the heart and left lung between mastectomy and BCS. Mean dose to the right lung was significantly less in mastectomy as compared to BCS, $0.29 \mathrm{~Gy} v$ s. $0.51 \mathrm{~Gy}$, respectively $(\mathrm{p}=0.007)$. Mean dose to the opposite breast was significantly lower in patients with mastectomy than BCS $(0.54 \mathrm{~Gy} V s 0.37 \mathrm{~Gy}, \mathrm{p}=0.007)$. The dose to the distal LAD was significantly higher than proximal LAD both in BCS (24.6Gy Vs $4.3 \mathrm{~Gy}, \mathrm{p}=<0.0001)$ and mastectomy (27.5Gy Vs $2.7 \mathrm{~Gy}, \mathrm{p}=<0.0001)$ patients.

Conclusion: There was no difference in doses received by heart and left lung between BCS and mastectomy patients. Mean doses to the right lung and breast were significantly less in mastectomy patients.
\end{abstract}

Key words: Breast cancer; Radiotherapy; BCS; mastectomy; heart dose

\section{Introduction}

During radiation delivery to breast or chest wall a part of the dose is also received by heart. Mean doses of radiation to the heart from radiotherapy for breast cancer are typically about 1-2Gy for disease of the right breast. For disease of the left breast the doses are usually higher but vary widely and for some women including those in whom the distance of the heart to the thoracic wall is small and those who require 
internal mammary radiation, the mean doses may be around 10Gy (Darby et al., 2013). Majority of studies reported in the literature are with conventional fraction (Aznar et al., 2011; Correa et al., 2008; Jin et al., 2013; Krueger et al., 2004; Mulliez et al., 2013; Muren et al., 2002; Nilsson et al., 2012; Remouchamps et al., 2003; Stewart et al., 2008; Swanson et al., 2013; Taylor et al., 2004). Hypofractionation is becoming a new standard in breast cancer irradiation. Hypofractionation is well established in WBI but it's acceptance in PMRT is still very low. There is a concern that heart may receive more dose after mastectomy than BCS in patients with left breast cancer and it is one of the reasons for low acceptance of hypofractionation after mastectomy. The dose to the LAD artery is greater than the dose to the whole heart. Left sided breast cancer had a statistically significant increase in rate of stenosis in the coronary artery branches on the left anterior surface of the heart [mid, distal and distal diagonal branch of the LAD coronary artery].

In this prospective study we compared dose to the heart, LAD artery, and bilateral lungs and opposite breast between mastectomy and BCS in patients with left sided breast cancer with hypofractionation. The null hypothesis was that OARs receive higher dose after mastectomy in patients with left breast cancer.

\section{Methods}

A total of 30 patients of left side breast cancer after mastectomy or BCS, 15 each were included in the study. Inclusion criteria were the primary cancer of the left breast of any histological type, age 2070 years, no metastasis and total mastectomy/BCS with resection margin free of tumour. Exclusion criteria were any history of prior malignancy, history of prior irradiation to the chest, pregnant or lactating women and collagen vascular disorders.

A CT based three dimensional planning using $100 \mathrm{ml}$ of IV contrast was used to reconstruct radiotherapy target volume for treating breast/ chest and loco regional lymph nodes. The patients were positioned supine on a breast board with arm abducted above the head on arm rest in planning CT in radiotherapy department. CT axial cuts were taken from the level of larynx to upper abdomen, including both the lungs with a scan thickness and index of $3 \mathrm{~mm}$. CT images were transferred to the Treatment Planning system (TPS). The chest wall or whole breast, heart, bilateral lungs and opposite breast were contoured using RTOG contouring guidelines. For LAD contouring, left coronary artery was identified and its course was followed. LAD was contoured from the place where it branches away from the main left coronary artery, and then runs towards the front and down towards the heart apex. It was divided in to proximal and distal parts. Distal LAD was the part which lies very near to the chest wall toward the apex of the heart. No additional margin was given to LAD.

The treatment parameters, patients and OARs outlines were exported to computerised TPS and plans were made using standard tangent fields (Figure 1a \& b). Dose prescribed was $40 \mathrm{~Gy} / 16 \# / 3$ weeks both in

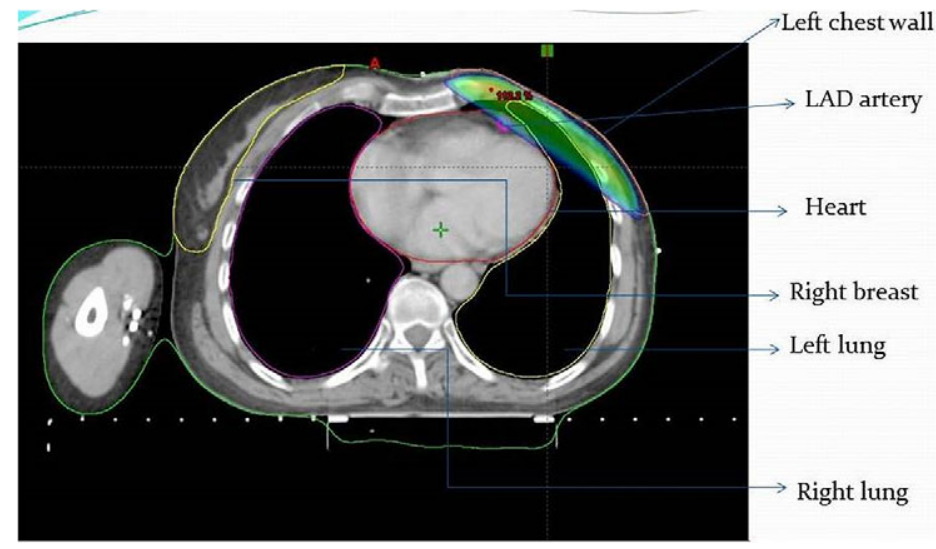

Figure 1a. Axial CT showing planning of patient after mastectomy 


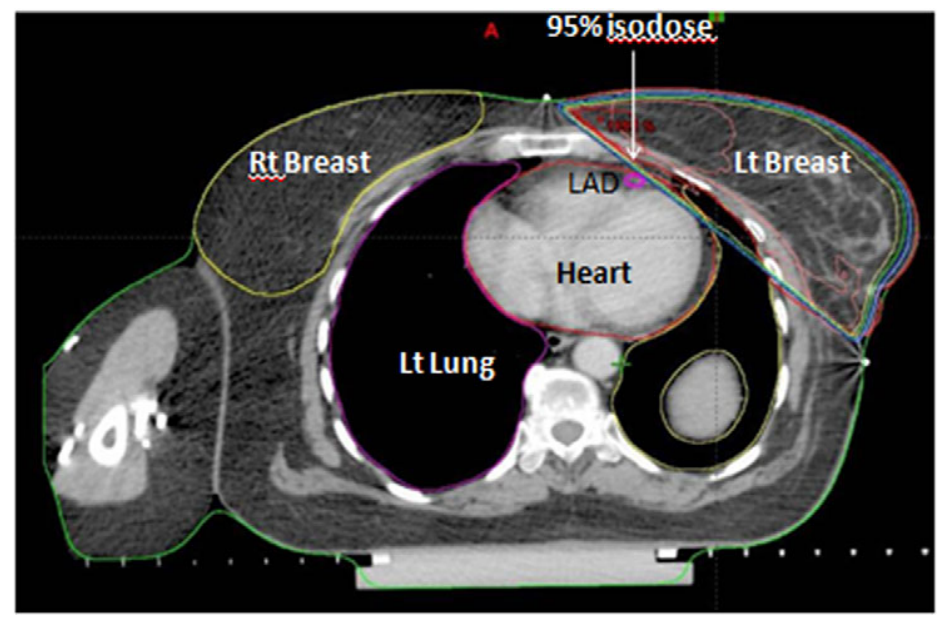

Figure 1b. Axial CT showing radiotherapy planning of BCS patient

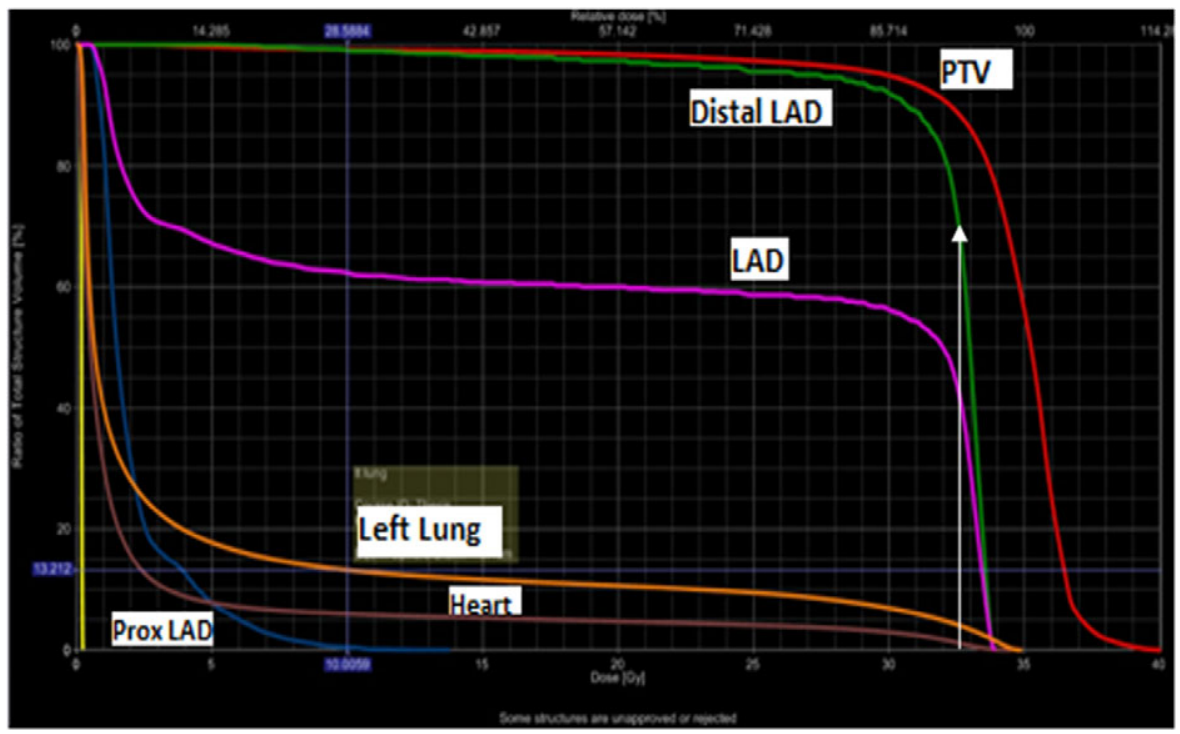

Figure 2. DVH showing dose received by different volume of OARs ( $70 \%$ of distal LAD is receiving $100 \%$ dose, white arrow)

BCS and mastectomy patients. Heart, bilateral lungs, LAD artery and opposite breast dose volume histogram were generated (Figure 2). From these, estimate of mean doses to heart, LAD, proximal LAD, distal LAD, bilateral lung and opposite breast, V5 of right lung, V5, V10 and V20 of left lung and V2 opposite breast were calculated and compared between BCS and mastectomy patients using student's ' $t$ ' test.

\section{Results}

Mean age of the patients was 45 years(22-67 years). Total 15(50\%) patients were premenopausal. Obesity was present in 5(16.6\%) patients and $12(40 \%)$ patients were overweight. 15(50\%) of patients had locally advanced disease and $17(57 \%)$ patients had positive nodes. Chemotherapy was given to $27(90 \%)$ patients, anthacyclins in $2(7 \%)$ and anthacyclins and taxanes in $25(93 \%)$ patients. Oestrogen receptor(ER) and 
progesterone receptors(PR) were positive in $12(40 \%), 7(23 \%)$ were only ER positive. 7(23\%) were Her2neu positive and only one received trastuzumab.

Mean doses to the heart, LAD, proximal LAD and distal LAD were 3.364Gy, 16.06Gy, 2.7Gy, 27.5Gy; and 4.219Gy, 14.653Gy, 4.306Gy, 24.6Gy, respectively for mastectomy and BCS patients (Table 1). There was no statistical significant difference in the doses to the heart between mastectomy and BCS patients.

Left lung mean dose, V5, V10 and V20 were 5.96Gy, 16\%, 14\%, 12.4\%; and 7.69Gy, 21\%, 18\%, 16\% in mastectomy and BCS patients, respectively. These were also not statistically different between the two techniques (Table 2).

Mean dose to the right lung was significantly less in mastectomy as compared to BCS patients, $0.29 \mathrm{~Gy}$ vs. $0.51 \mathrm{~Gy}$, respectively $(\mathrm{p}=0.007)$.

Mean dose to the opposite breast was significantly higher in patients with BCS than mastectomy (0.54Gy Vs $0.37 \mathrm{~Gy}, \mathrm{p}=0.007)$ as shown in Table 2 .

Similarly V2 to the right breast was also higher in BCS $1.43 \%$ as compared to $0.26 \%$ in mastectomy patients but it was not statistically significant $[(\mathrm{p}=0.07)$ Table 2$]$.

The dose to the distal LAD was significantly higher than proximal LAD both in BCS (24.6Gy Vs 4.3Gy, $\mathrm{p}=<0.0001)$ and mastectomy $(27.5 \mathrm{~Gy} V s 2.7 \mathrm{~Gy}, \mathrm{p}=<0.0001)$ patients (Table 3). It can be seen in Figure 2 that $70 \%$ of distal LAD is receiving $100 \%$ dose (white arrow).

\section{Discussion}

In this dosimetric comparative study between BCS and mastectomy patients with left breast cancer there was no significant difference in dose to the heart, LAD, proximal LAD and distal LAD. Mean dose to the heart was 3.364Gy and 4.219Gy for mastectomy and BCS patients, respectively. In this study we used hypofractionated radiotherapy schedule of $40 \mathrm{~Gy} / 16 \# / 3$ weeks both in BCS and mastectomy patients.

Cardiac dose due to breast radiotherapy has been reported in many studies (Table 3) (Arenas et al., 2014; Awad et al., 2013; Borca et al., 2012; Darby et al., 2013; Gursel et al., 2011; Pili et al., 2011; Schubert et al., 2011; Tan et al., 2011; Yavas et al., 2012). In majority of these studies conventional fractionation of 50Gy/25\#/5 weeks with or without boost was used (Arenas et al., 2014; Awad et al., 2013; Borca et al., 2012; Gursel et al., 2011; Khullar et al., 2014; Schubert et al., 2011; Tan et al., 2011; Yavas et al., 2012) with few studies with hypofraction (Pili et al., 2011). We did not come across any study in the English literature where a comparison has been done for doses to the OARs between mastectomy and BCS in patients with left breast cancer. Mean dose to the heart was comparable between mastectomy and BCS. The reason for comparable dose in the present study could be the contouring guideline and different structures included for the whole breast irradiation(WBI) and chest wall irradiation(CWI) in these patients. For WBI the entire breast volume is contoured excluding $3-5 \mathrm{~mm}$ of the skin and pectoral muscles to constitute clinical target volume(CTV). For CWI skin, pectoral, chest wall muscles and ribs are included in the CTV. So heart and the left lung are very close to the CTV. For contouring whole breast, the breast borders are variable and depend on the extent of the breast. These variable borders invariably lead to a variable radiation volume to be treated in case of WBI and variable dose to the OARs. Whereas in PMRT, the chest wall borders or land marks are well defined and volume of irradiation is also well defined.

Cardiac RT dose reported in the literature vary with techniques, prescribed dose and the period in which patients were treated. Higher mean cardiac dose reported by Darby et al. was due to the inclusion of the patients from 1960-70s and retrospective analysis of dose on the basis of CT scans of a patient of typical anatomy (one size for all) which may not be representative of all population (Darby et al., 2013). Higher mean cardiac doses of 7Gy in 1970s and 5Gy in 1980s were reported by Hooning et al. (2007) and Taylor et al. (2007) (10.5Gy in 1970s). These were due to the older technique of radiation from 1950-1980 and compulsory internal mammary irradiation at that time. Capezzali et al. reported lower cardiac dose $(1.71 \mathrm{~Gy})$ in their study. It was due to lateral decubitous treatment position in which the breast falls away from the chest wall (Capezzali et al., 2013). This treatment technique is not generally followed because of set up uncertainty. Higher doses to the OARs in other studies were due to the higher prescription dose to the PTV (50-62.4Gy) (Assaoui et al., 2012; Awad et al., 2013; Guan et al., 2015; Ma et al., 2015; Pierce 
et al., 2002; Tan et al., 2011; Yavas et al., 2012). We planned all our patients in supine position on breast board and used hypofractionation in all our patients. Some of the studies have also included patients with internal mammary irradiation which also contribute to higher doses to the OARs (Hjelstuen et al., 2012; Khullar et al., 2014; Zhang et al., 2015). We did not plan internal mammary radiation in this study. Since these older techniques and conventional schedules are not followed now so there was a need to know the doses to the OARs with hypofractionation which is becoming standard of care in adjuvant breast/chest wall irradiation.

Because of its anatomical location, LAD artery is likely to remain within the high dose volume from a tangential field arrangement even at low irradiated heart volume and is most prone for atherosclerosis (Figure 2). Few studies has reported dose received by LAD where it ranged from 0.1 to $46 \mathrm{~Gy}$ (Gursel et al., 2011; Ma et al., 2015; Muren et al., 2002; Taylor et al., 2004; 2007; Yavas et al., 2012). In our study dose to the LAD was $14.6 \mathrm{~Gy}$ and $16 \mathrm{~Gy}$ in patients with BCS and mastectomy, respectively. Very few studies have reported dose to the proximal LAD, where it ranged from 12-17.8Gy (Aznar et al., 2011; Krueger et al., 2004). Only one study had reported dose to the distal LAD where it was 31.52Gy (Krueger et al., 2004). In the present study doses to proximal and distal LAD were lower as compared to other studies. It might be because of lower total dose used in our study with hypofractionation. With hypofractionation the total dose is reduced to compensate for high dose per fraction but it is biologically equivalent to the conventional fractionation. So with this equally effective dose, the dose to the OARs is reduced. A very low dose to the whole LAD artery seems to be associated with very low dose to the heart but the reverse statement is not true. That is why the whole LAD artery should be contoured as a risk organ along with the whole heart and should be used prospectively for plan optimism in left sided breast cancer. In our study dose to the distal LAD was significantly higher than the proximal LAD in both BCS and mastectomy patients (Fig 2). Distal LAD dose may be of paramount importance as it may contribute to the late effects on the heart. So it is important to report dose received by distal LAD artery.

In our study, there was no difference in mean dose, V5, V10 and V20 of left lung between BCS and mastectomy patients. It also confirms that hypofractionation can be practiced in patients with mastectomy for left breast cancer without concerns for late effects because these patients are likely to develop late toxicity comparable to those with WBI for which hypofractionation is well established. In a study by Remouchamps et al. (2003) V20 to left lung was 17.1 to $22.3 \%$, which is higher than our and the other studies reported in the literature where it mostly ranged from 8.2 to 14.8\% (Borca et al., 2012; Gursel et al., 2011; Schubert et al., 2011; Ng et al., 2012).

In the present study no volume of right lung received 5Gy in both BCS and mastectomy patients because plans were made using standard tangent fields, so the chances of dose going right lung were very remote. Only two studies have reported mean dose to the right lung where it ranged from 0.1 to $0.8 \mathrm{~Gy}$ (Schubert et al., 2011; Tan et al., 2011). In the present study, mean dose to the right lung was 0.51Gy and $0.29 \mathrm{~Gy}$ in patients with BCS and mastectomy, respectively. This is comparable to those reported in other studies.

Mean dose to the contralateral breast was higher in patients with BCS as compared to mastectomy in the present study. Rather et al. reported dose to the contra lateral breast of $5.34-6.40 \%$ of the total dose for tangential fields and it was $1.2-1.75 \%$ of the dose for supraclavicular fields in their study (Rather et al., 2014). Larger dose to contra lateral breast may be because of short perpendicular distance from the contra lateral breast surface to the geometric beam edge. Short perpendicular distance can be caused by shallow medial gantry angle or large pendulous breast. Higher mean dose to the right breast in our study can be explained by scattering from wedges used for compensation in BCS patients.

Limitation of the present study are small sample size for very variable patient group, bias inherent to the trial's design, conclusions only apply to the specific technique used, variety anatomical background of the heart, and absence of clinical outcomes as this being only a dosimetric study. Boost in cases of BCS patients was not planned in the present study, which may also contribute doses to the OARs.

Based on the present study and other studies from the literature it can be concluded that the dose to the OARs varies from patient to patient depending upon the patient anatomy, indication (post mastectomy/ $\mathrm{BCS}$ ), contouring guidelines for various volumes and organ at risks (OARs), radiation technique, volume 
of treatment, dose and nature of study (prospective/retrospective). However, as hypofractionation is going to be the standard for breast radiotherapy, therefore if same dose fractionation is used in patients with BCS or after mastectomy doses to the OAR may not vary.

\section{Conclusion}

In the present study there was no difference between dose received by heart, LAD, proximal LAD and distal LAD; mean dose, V5, V10 and V20 of left lung between BCS and mastectomy patients. Mean dose to the contra lateral breast was higher in BCS patients. Therefore hypofractionation may be safely delivered in patients with left side breast cancer after mastectomy. It will contribute to increase the utilization of hypofractionation in PMRT setting.

Compliance with Ethical Standards.

Funding. None.

Conflicts of interest None.

Ethical approval. All procedures performed in studies involving human participants were in accordance with the ethical standards of the institutional and/or national research committee and with the 1964 Helsinki declaration and its later amendments or comparable ethical standards.

Research involved. human participants.

Informed consent. Informed consent was obtained from all individual participants included in the study.

Data availability. Data is available.

Authors' contributions. Dr Budhi Singh Yadav: Concept design, Treatment planning, data collection and analysis, manuscript writing and approval.

Dr Deepak Kumar Das: Concept design, Treatment planning, data collection, analysis and approval.

Dr Narendra Kumar: Concept design, manuscript approval.

Dr Manphool Singhal: Concept design, manuscript approval.

Dr Ngangom Robert: Treatment planning, and approval.

Conflict of interest statements. None.

Role of funding source. None.

Ethics committee approval. Yes

Supplementary Materials. To view supplementary material for this article, please visit http://dx.doi.org/10.1017/exp.2021.8.

\section{References}

Arenas, M., Hernandez, V., Farrus, B., Muller, K., Gascon, M., Pardo, A., et al. (2014). Do breast cancer improve breast cancer dosimetry? A comparative study for patients with large or pendulous breasts. Acta Oncologica, 53, 795-801.

Assaoui, F., Toulba, A., Nouh, M., Lkhouyaali, S., Bensouda, Y., Kebdani, T., et al. (2012). Mono-isocentric technique in the breast cancer and organ at risk tolerance. Journal of Nuclear Medicine \& Radiation Therapy, S2 DOI: 10.4172/2155-9619.S2010 .

Awad, I., Zayed, D. H., Abotouk, N. A., \& Dawod, T. (2013). Moving from 2D to 3D-CRT planning of chest wall for post mastectomy breast cancer patients: Mansoura University experience. The Medical Journal of Cairo University, 81, 21-27.

Aznar, M. C., Korreman, S. S., Pedersen, A. N., Josipovic, M., \& Specht, L. (2011). Evaluation of dose to cardiac structures during breast irradiation. British Journal of Radiology, 84, 743-746.

Borca, V. C., Franco, P., Catuzzo, P., Migliaccio, F., Zenone, F., Aimonetto, S., et al. (2012). Does tomodirect 3D CRT represent a suitable option for post-operative whole breast irradiation? A hypothesis-generating pilot study. Radiation Oncology, 7, 211. 
Capezzali, G., Kirova, Y. M., Costa, E., Fournier-Bidoz, N., Aristei, C., Zygogianni, A., et al. (2013). Left breast cancer treated in isocentric lateral decubitus (ILD) position: An alternative technique sparing organs at risk (OAR). Global Journal of Breast Cancer Research, 1, 53-57.

Correa, C. R., Das, I. J., Litt, H. I., Ferrari, V., Hwang, W.-T., Solin, L. J., \& Harris, E. E. (2008). Association between tangential beam treatment parametres and cardiac abnormalities after definitive radiation treatment for left sided breast cancer. International Journal of Radiation Oncology, Biology, Physics, 72, 508-516.

Darby, S. C., Ewertz, M., McGale, P., Bennet, A. M., Blom-Goldman, U., Bronnum, D., et al. (2013). Risk of ischemic heart disease in women after radiotherapy for breast cancer. NEJM, 368, 987-998.

Guan, H., Dong, L., Ding, L., Zhang, Z., Huang, W., Liu, C., et al. (2015). Morphological factors and cardiac doses in whole breast radiation for left-sided breast cancer. Asian Pacific Journal of Cancer Prevention, 16, 2889-2894.

Gursel, B., Meydan, B., Ozbek, N., \& Ofluoglu, T. (2011). Dosimetric comparison of three different external beam whole breast irradiation techniques. Advances in Therapy, 28, 1114-1125.

Hjelstuen, M. H. B., Mjaaland, I., Vikstrom, J., \& Dybvik, I. (2012). Radiation during deep inspiration allows loco regional treatment of left breast and axillary, supraclavicular and internal mammary lymph nodes without compromising target coverage or dose restrictions to organs at risk. Acta Oncologica, 51, 333-344.

Hooning, M. J., Botma, A., Aleman, B. M. P., Baaijens, M. H. A., Bartelink, H., Klijn, J. G. M., et al. (2007). Long term survivors of cardiovascular disease in 10-year survivors of breast cancer. Journal of the National Cancer Institute, 99, 365-375.

Jin, G., Chen, L., Deng, X., Liu, X., Huang, Y., \& Huang, X. (2013). A comparative dosimetric study for treating left-sided breast cancer for small breast size using five different radiotherapy techniques: conventional tangent field, field in field, tangential-IMRT, Multi-beam IMRT and VMAT. Radiation Oncology, 8, 89.

Khullar, P., Datta, N. R., Vekadamanickam, G., Garg, C., \& Sinha, S. (2014). Comparative dosimetric evaluation of intensity modulated radiation therapy versus conventional radiotherapy in postoperative radiotherapy of breast cancer. Journal of Nuclear Medicine \& Radiation Therapy, 5, 4.

Krueger, E. A., Schipper, M. J., Koelling, T., Marsh, R. B., Butler, J. B., \& Pierce, L. J. (2004). Cardiac chamber and coronary artery doses associated with post mastectomy radiotherapy techniques to the chest wall and regional nodes. International Journal of Radiation Oncology, Biology, Physics, 60, 1195-1203.

Ma, C., Zhang, W., Lu, J., Wu, L., Wu, F., Huang, B., et al. (2015). Dosimetric comparison and evaluation of three radiotherapy techniques for use after modified radical mastectomy for locally advanced left-sided breast cancer. Scientific Reports, 21, 12274.

Mulliez, T., Speleers, B., Madani, I., Gersem, W. D., Veldeman, L., \& Neve, W. D. (2013). Whole breast radiotherapy in prone and supine position: Is there a place for multi-beam IMRT?. Radiation Oncology, 8, 151.

Muren, L. P., Maurstad, G., Hafslund, R., Anker, G., \& Dahl, O. (2002). Cardiac and pulmonary doses and complication probabilities in standard and conformal tangential irradiation in conservative management of breast cancer. Radiotherapy and Oncology, 62, 173-183.

Ng, J., Shuryak, I., Xu, Y., Chao, K. S. C., Brenner, D. J., \& Burri, R. J. (2012). Predicting the risks of secondary lung malignancies associated with whole-breast radiotherapy. International Journal of Radiation Oncology, Biology, Physics, 83, $1101-1106$.

Nilsson, G., Holmberg, L., Garmo, H., Duvernoy, O., Sjogren, I., Lagerqvist, B., \& Blomqvist, C. (2012). Distribution of coronary artery stenosis after radiation for breast cancer. Journal of Clinical Oncology, 30, 380-386.

Pierce, L. J., Butler, J. B., Martel, M. K., Normolle, D. P., Koelling, T., Marsh, R. B., et al. (2002). Post mastectomy radiotherapy of the chest wall: Dosimetric comparison of common techniques. International Journal of Radiation Oncology, Biology, Physics, 52, 1220-1230.

Pili, G., Grimaldi, L., Fidanza, C., Florio, E. T., Petruzzelli, M. F., D’Errico, M. P., et al. (2011). Geometric and dosimetric approach to determine probability of late cardiac mortality in left tangential breast irradiation: Comparison between wedged beams and field-in-field technique. International Journal of Radiation Oncology, Biology, Physics, 81, 894-900.

Rather, S. A., Haq, M. M., Khan, N. A., Khan, A. A., \& Sofi, A. G. (2014). Determining the contra lateral breast dose during radiotherapy of breast cancer using rainbow dosimeter. Journal of Radiation Research and Applied Sciences, 7, 384-389.

Remouchamps, V. M., Vicini, F. A., Sharpe, M. B., kestin, L. L., Martinez, A. A., \& Wong, J. W. (2003). Significant reduction in heart and lung doses using deep inspiration breath hold with active breathing control and intensity-modulated radiotherapy for patients treated with loco regional breast irradiation. International Journal of Radiation Oncology, Biology, Physics, 55, 392-406.

Schubert, L. K., Gondi, V., Sengbusch, E., Westerly, D. C., Soisson, E. T., Paliwal, B. R., et al. (2011). Dosimetric comparison of whole breast irradiation with 3D CRT, forward-planned IMRT, inverse-planned IMRT, helical tomotherapy and topotherapy. Radiotherapy and Oncology, 100, 241-246.

Stewart, A. J., O’Farrell, D. A., Cormack, R. A., Hansen, J. L., Khan, A. J., Mutyala, S., et al. (2008). Dose volume histogram analysis of normal structures associated with accelerated breast irradiation delivered by high dose rate brachytherapy and comparison with whole breast external beam radiotherapy fields. Radiation Oncology, 3, 39. 
Swanson, T., Grills, I., Ye, H., Teahan, M., Letts, N., Yan, D., et al. (2013). Six-year experience routinely utilizing moderate deep inspiration breath hold ( $\mathrm{mDIBH})$ for the reduction of cardiac dose in left-sided breast irradiation for patients with early stage or locally advanced breast cancer. American Journal of Clinical Oncology, 36, 24-30.

Tan, W., Wang, X., Qiu, D., Liu, D., Jia, S., Zeng, F., et al. (2011). Dosimetric comparison of intensity-modulated radiotherapy plans, with or without anterior myocardial territory and left ventricle as organs at risk, in early-stage left-sided breast cancer patients. International Journal of Radiation Oncology, Biology, Physics, 81, 1544-1551.

Taylor, C. W., Nisbet, A., McGale, P., \& Darby, S. C. (2007). Cardiac exposures in breast cancer radiotherapy: 1950s-1970s. International Journal of Radiation Oncology, Biology, Physics, 69, 1484-1495.

Taylor, C. W., Povall, J. M., McGale, P., Nisbet, A., Dodwell, D., Smith, J. T., \& Darby, S. C. (2004). Cardiac dose from tangential breast cancer radiotherapy in the year 2006. International Journal of Radiation Oncology, Biology, Physics, 72, 501-507.

Yavas, G., Yavas, C., \& Acar, H. (2012). Dosimetric comparison of whole breast radiotherapy using field in field and conformal radiotherapy techniques in early stage breast cancer. Iranian Journal of Radiation Research, 10, 131-138.

Zhang, L., Mei, X., Chen, X., Hu, W., Hu, S., Zhang, Y., et al. (2015). Estimating cardiac substructures exposure from diverse radiotherapy techniques in treating left-sided breast cancer. Medicine, $\mathbf{9 4}, 847$.

Cite this article: Yadav BS, Das DK, Kumar N, Singhal M, Robert N (2021). Radiation dose to the heart with hypofractionation in patients with left breast cancer Experimental Results, 2, e21, 1-11. https://doi.org/10.1017/exp.2021.8 


\title{
Peer Reviews
}

\section{Reviewing editor: Prof. Martin Michaelis}

University of Kent, School of Biosciences, Canterbury, United Kingdom of Great Britain and Northern Ireland, CT2 7NJ

This article has been accepted because it is deemed to be scientifically sound, has the correct controls, has appropriate methodology and is statistically valid, and has been sent for additional statistical evaluation and met required revisions.

doi:10.1017/exp.2021.8.pr1

Review 1: Radiation dose to the left anterior descending coronary artery in patients with left breast cancer

Reviewer: Dr. Pieter Deseyne

Ghent University Hospital, Radiation Oncology, Corneel Heymanslaan 10, Ghent, Belgium, 9000

Date of review: 19 April 2021

\begin{abstract}
(C) The Author(s), 2021. Published by Cambridge University Press. This is an Open Access article, distributed under the terms of the Creative Commons Attribution licence (http://creativecommons.org/licenses/by/4.0), which permits unrestricted re-use, distribution and reproduction, provided the original article is properly cited.
\end{abstract}

Conflict of interest statement. Reviewer declares none

Comments to the Author: I recommend substantial revisions. While the conclusions seem logical, they are impossible to reproduce given the limited methods section. Therefore the reader is unable to adequately assess the scientific soundness and validity of the methods. Please elaborate on your methods: retrospective vs prospective inclusion/planning? Nodal irradiation is mentioned and is a major contributing factor to OAR dose. Which contouring guidelines are used for targets and OARs? Please elaborate on beam design. Describe patient characteristics.

The reported OAR parameters are reported without a measure of spread, please include one. There is no way for the reader to know that OAR doses were obtained with adequate target volume coverage, please clarify. Maybe include a whole group mean DVH for all ROIs.

When comparing to other papers, please clarify which use hypofractionation. Given the many factors influencing OAR doses the authors cite in the discussion, it is surprising they don't clarify these factors for their current trial.

The article doesn't cite limitations but obviously has some (e.g. small sample size for very variable patient group, bias inherent to the trial's design, conclusions only apply to the specific technique used). Furthermore, the impact of a tumor bed boost is not discussed. It might well be that RT after BCS actually yields larger OAR doses than post mastectomy RT because of it.

The manuscript needs some language revisions but can be understood as is. Please explain abbreviations (e.g. TMAC). The title in the submission system (see proof p.1) doesn't match the one from the abstract. 
Is the data presented in the most useful manner? (40\%) 3/5

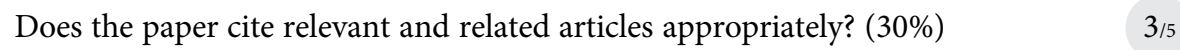

Context

Does the abstract correctly embody the content of the article? (25\%)

Does the introduction give appropriate context? (25\%)

Is the objective of the experiment clearly defined? $(25 \%)$

Analysis

Are the limitations of the experiment as well as the contributions of the experiment clearly outlined? (20\%) 


\title{
Review 2: Radiation dose to the left anterior descending coronary artery in patients with left breast cancer
}

Reviewer: Dr. Kazuhiko Sato

Tokyo West Tokushukai Hosp

Date of review: 30 April 2021

\begin{abstract}
(c) The Author(s), 2021. Published by Cambridge University Press. This is an Open Access article, distributed under the terms of the Creative Commons Attribution licence (http://creativecommons.org/licenses/by/4.0), which permits unrestricted re-use, distribution and reproduction, provided the original article is properly cited.
\end{abstract}

Conflict of interest statement. Reviewer declares none

Comments to the Author: Authors addressed the safety of hypofractionated radiotherapy as PMRT after mastectomy compared with whole-breast irradiation after BCS. The topic is relevant and their conclusions were solid. Because of small number of patients, authors should highlight on the heart dose in the hypofractionation schedules. The title of this article is "Comparison of radiation dose to the organs at risk after breast conservation surgery and mastectomy in patients with left breast cancer." However, there should be the words of "heart" and "hypofractionation." In the materials and methods, the detailed contouring method of the LAD, etc. should be described. In the discussion, the limitations of this study should be introduced, such as small sample size, variety anatomical background of the heart, and absence of clinical outcomes.

\section{Score Card}

Presentation

3.0

Is the article written in clear and proper English? (30\%)

Is the data presented in the most useful manner? (40\%)

Does the paper cite relevant and related articles appropriately? (30\%)

\section{Context}

3.0

Does the title suitably represent the article? (25\%)

Does the abstract correctly embody the content of the article? (25\%)

Does the introduction give appropriate context? (25\%)

Is the objective of the experiment clearly defined? (25\%)

Analysis

Are the limitations of the experiment as well as the contributions of the experiment clearly outlined? $(20 \%)$ 